\title{
Одноэлектронный эмиссионно-инжекционный транспорт в микроструктуре с коллоидными квантовыми точками узкозонных полупроводников
}

\author{
(С) Н.Д. Жуков ${ }^{1}$, М.В. Гавриков ${ }^{1,2}$, В.Ф. Кабанов ${ }^{2}$, И.Т. Ягудин ${ }^{1}$ \\ ${ }^{1} \mathrm{OOO}$ „НПП Волга“, \\ 410033 Саратов, Россия \\ ${ }^{2}$ Саратовский государственный университет им. Н.Г. Чернышевского, \\ 410012 Саратов, Россия \\ E-mail: ndzhukov@rambler.ru
}

Поступила в Редакцию 16 ноября 2020 г.

В окончательной редакции 8 декабря 2020 г.

Принята к публикации 18 декабря 2020 г.

\begin{abstract}
Методом аппроксимации вольт-амперных характеристик коллоидных квантовых точек узкозонных полупроводников $\mathrm{InSb}$ и $\mathrm{PbS}$ показано, что в одноэлектронном режиме транспорт электронов в разных интервалах напряжения определяется одним из конкурирующих процессов - эмиссией из квантовой точки, инжекцией в нее и пролетом в ней с ограничением тока пространственным зарядом. При напряжении $>0.5 \mathrm{~B}$ для одиночных квантовых точек на вольт-амперных характеристиках наблюдались участки нестабильности и провала тока, подобные кулоновской щели. Качественные и числовые сравнительные оценки позволяют считать, что в структуре сегрегированного множества квантовых точек наблюдаются одноэлектронный транспорт и ограничение тока, подобное кулоновской блокаде. Воздействие светом при измерении вольтамперных характеристик срывает или усиливает эффект, увеличивая или уменьшая ток в зависимости от спектра возбуждающего излучения.
\end{abstract}

Ключевые слова: коллоидная квантовая точка, одноэлектронный транспорт, электронная эмиссия, электронная инжекция, конкурирующий электронный процесс, ограничение тока пространственным зарядом, кулоновская блокада, кулоновская щель.

DOI: $10.21883 /$ FTP.2021.04.50732.9552

\section{1. Введение}

Базовыми элементами перспективных направлений наноэлектроники являются квантово-размерные структуры, в частности квантовые точки (КТ), формируемые двумя вариантами нанотехнологий: „сверху“ - островковой литографией по эпитаксиальному слою; „снизу“ - самосинтезом в термодинамически равновесной среде. По второму варианту наиболее распространенным является коллоидный синтез.

Базовым физическим процессом в квантовых точках является одноэлектронный транспорт $[1,2]$. Соответственно, такие наноструктуры называют одноэлектронными, а направление науки и техники - одноэлектроникой [3].

В КТ металлов и широкозонных полупроводников электроны последовательно туннелируют через квантовую точку, что возможно, когда размер КТ - не более $1-2$ нм. Квантовые точки узкозонных полупроводников имеют высокие параметры размерного квантования, что позволяет наблюдать квантово-размерные эффекты в случаях их относительно больших размеров. Размеры КТ при этом должны быть не более длины волны де Бройля для электрона $\lambda=h(2 m \epsilon)^{-1 / 2}(h-$ постоянная Планка, $m-$ эффективная масса электрона, $\epsilon-$ его кинетическая энергия).

В отличие от КТ с размерами 1-2нм, в квантовых точках больших размеров прямое туннелирование через нее мало вероятно. В этом случае транспорт электрона будет состоять, последовательно, из процессов его инжекции в КТ, термализации его состояний и транспорта в ней, эмиссии из КТ.

Квантовая точка для электрона является глубокой потенциальной ямой, что приводит к ее специфическому свойству блокирования переноса последующего электрона предыдущим, названному кулоновской блокадой [1-4]. На ВАХ при этом образуется специфический провал, названный кулоновским, и участок отрицательного сопротивления. Поскольку электронный ток неизбежно связан с перезарядкой наноконденсато$\mathrm{pa}$, возникают осцилляции тока. Этот процесс широко изучен для одиночных КТ и мало - для их ансамблей в микроструктуре. По данному вопросу имеется обширная литература [5-7], но исследования в основном посвящены квантовым точкам на базе материалов с относительно невысокими параметрами размерного квантования, когда практически легко наблюдаются специфические эффекты, такие как, например, кулоновская блокада.

Детальное исследование этих процессов имеет важное значение для формирования таких элементов наноэлектроники, как одноэлектронный транзистор [8] и элементы нанооптоэлектроники [9].

В нашей работе, в продолжение $[10,11]$, в более широком аспекте образцов, измерений и анализа исследованы свойства одноэлектронного транспорта в микроструктуpax двух вариантов с коллоидными квантовыми точками 
узкозонных полупроводников антимонида индия и сульфида свинца: $\mathrm{InSb}, \mathrm{InSb}(\mathrm{InP}-\mathrm{CdS}), \mathrm{PbS}, \mathrm{PbS}(\mathrm{CdS})$. Для сравнительного анализа использованы квантовые точки относительно широкозонного полупроводника селенида кадмия - $\mathrm{CdSe}(\mathrm{CdS})$. Во всех случаях в скобках указаны химические формулы оболочек КТ.

\section{2. Методика исследования}

Коллоидные квантовые точки синтезированы по технологии, описанной в работах $[12,13]$. Качество полученных КТ контролировалось на спектрофлуориметpe Fluorolog (Horiba), спектрофотометре Cary 5000 (Varian), просвечивающем Libra-120 и растровом Mira II LMU электронных микроскопах. Размеры наночастиц $d$ измерялись методом динамического светорассеяния с помощью прибора Malvern Zetasizer и электронно-микроскопического ТЕМ-исследования на просвечивающем микроскопе, на котором также определяли и форму квантовых точек.

На рис. 1 представлена ТЕМ-фотография квантовых точек $\mathrm{InSb}(\mathrm{InP} / \mathrm{CdS})$, где видно, что форма КТ преимущественно квадратная и треугольная. Это может означать, что рост кристаллитов при коллоидном самосинтезе происходил в направлениях, перпендикулярных плоскостям с минимальной энергией связи [100] или [111], и, таким образом, в данном случае удалось синтезировать КТ совершенной кристаллической структуры. Кроме того, по ТЕМ-картинке можно судить о характере соединения наночастиц между собой - c зазорами нанометрового и субнанометрового размеров.

Квантовые точки наносились на подложки с микроструктурой разработанных нами двух вариантов: по технологии Ленгмюра-Блоджетт [14], для исследований одиночных КТ, и планарной технологии $[11]$ - для ансамблей КТ.

На сканирующем зондовом микроскопе (С3М) исследованы монослои квантовых точек, сформированные на поверхности водной субфазы по технологии Ленгмюра-Блоджетт и перенесенные на твердые подложки с проводящим слоем индий-оловянного оксида (ITO). Исследования электрофизических свойств полученных образцов с квантовыми точками проводились с помощью микроскопа C3M SOLVER Nano.

Перед исследованием вольт-амперной характеристики (BAX - функциональная зависимость тока $I$ от напряжения $V$ ) отдельной наночастицы проводилось сканирование поверхности пленки методами сканирующей туннельной микроскопии (СТМ) в режиме стабилизированного тока. После анализа полученного СТМобраза поверхности макрообразца на нем выбиралось не менее 10 точек для снятия ВАХ в автоматическом режиме, не менее 20 - на точку. По воспроизводимости результатов измерений отбирались точки с устойчивыми характеристиками, после чего проводились усреднения измеренных BAX. Результаты измерений обрабатывались по общепринятым методикам сканирующей зондовой микроскопии полупроводниковых наноструктур [15].

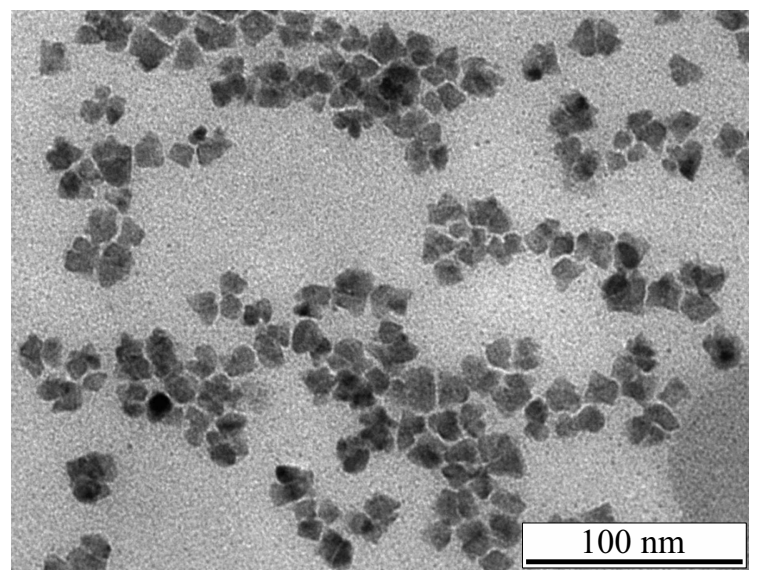

Рис. 1. ТЕМ-снимок квантовых точек антимонида индия.

Для гарантированного наблюдения эмиссионноинжекционного механизма тока измерения проводились в относительно широком интервале величин нанозазора $d_{0}$ (до 15 нм) зонд-образец, который обеспечивался манипуляциями положения зонда и заданием определенных значений тока и напряжения. Контроль величин зазора $d_{0}$ проводился по ВАХ эмиссии из зонда методом, описанным в наших работах $[16,17]$.

Все указанные меры при исследованиях на нашем невакуумном сканирующем туннельном микроскопе защищали от влияния нежелательных явлений и получения неадекватных результатов (артефактов).

Исследованы свойства ансамблей квантовых точек в микроструктуре, описанной в нашей работе [11]. Измерения BAX проводились с использованием нестандартного генератора однополярного низкочастотного импульсного „Пилообразного“ напряжения. Предварительно проводились измерения на микроструктуре без квантовых точек на предмет определения величин напряжения пробоя и паразитных утечек. Кроме того, проводилась оценка величин сопротивлений контакта металла электрода с используемыми в работе полупроводниками по методу, описанному в нашей работе [16].

Анализ ВАХ проводился методом построения кривых в разных координатах с использованием программы Excel и сопоставления величин достоверности аппроксимации. При этом для определения модельных механизмов тока использованы построения в координатах $(\ln I \sim \ln V), \quad(\ln I \sim V), \quad(\ln I \sim 1 / V)$, как это принято для полупроводниковых структур [16-18]. Определялись средние по образцам значения параметров и отклонения от среднего. ВАХ при транспорте электронов на том или ином ее участке лимитируется одним из процессов - перезарядкой наноконденсатора, эмиссией электрона из КТ, инжекцией и пролетом электрона в КТ. Особое внимание уделено особенностям характеристик.

Одноэлектронный режим измерений обеспечивался ограниченными сверху величинами тока с учетом оцен- 
ки времени пролета электрона в микро-, наноструктуре. Сам процесс электронного транспорта в данном случае правильно назвать поэлектронным, а не одноэлектронным.

Наиболее важные данные сведены в таблице, в которой обозначены: $E_{g}-$ ширина запрещенной зоны полупроводника, а остальные расшифрованы в тексте.

\section{3. Результаты измерений и их обсуждение}

\section{1. ВАХ одиночных КТ (метод СЗМ)}

Всего было измерено в диапазонах тока до 50 нА и напряжения - до $1.5 \mathrm{~B}$ : на десяти вариантах образцов (см. таблицу), на каждом - по 15 точек, для каждой точки - по 4 группы BAX, каждая из которых отличалась величиной зазора $d_{0}$ между зондом и образцом от $\sim 1$ до $\sim 10$ нм. При этом до 75\% измерений имели хорошее функциональное (по виду и характеру ВАХ) воспроизведение. ВАХ во всех случаях измерений имели явно выраженные достоверно функционально аппроксимируемые участки.

На участке напряжения до $0.25 \mathrm{~B}$ ВАХ первой группы измерений $\left(d_{0} \sim 1 \mathrm{Hм}\right)$ для обеих полярностей на образце имели зависимость для всех образцов с высокой степенью достоверности аппроксимации (не хуже $0.995)$, соответственно, формуле $I \sim R^{-1} V^{m}$. При этом $m=1 \pm 0.05$. Найденные из кривых средние для каждого образца значения $R$ были в пределах (0.09-0.15) МОм. Для измерений ВАХ групп 2-4 и полярности „плюс“ на образце наблюдалась та же зависимость, кроме того, что для полярности „минус“ на образце $-m \sim 1.25$.

Эквивалентную схему измеряемых образцов можно представить как замкнутый через источник питания контур последовательно соединенных емкости $C$ и сопротивления $R$. Ток в цепи при условии поэлектронной перезарядки наноконденсатора: $I \sim q(R C)^{-1}$, и тогда можно определить примерные величины емкости: $C_{Q 0} \sim q(R I)^{-1}=q / V_{Q 0}$, где за $V_{Q 0}$ принято наибольшее значение напряжения при соблюдении омического характера BAX, $q$ - заряд электрона. Рассчитанные таким образом значения емкости наноконденсатора были в пределах $(4.5-6.1) \cdot 10^{-19} \Phi$ и практически мало зависели от параметров квантовых точек, в частности, таких как значительно отличающиеся величины диэлектрической проницаемости $\varepsilon / \varepsilon_{0}$ и параметры размерного квантования $\Lambda, \Lambda / d$. Это может свидетельствовать о том, что определяющий вклад в емкость дает последовательно соединенный с самой квантовой точкой наноконденсатор зазора $d_{0}$ между зондом и КТ. По измерениям было установлено также, что с увеличением зазора $d_{0}$ найденные по BAX значения емкости, как и должно быть, уменьшались.

С увеличением напряжения $>0.25 \mathrm{~B}$ картина ВАХ принципиально изменялась. Для полярности „плюс“ на
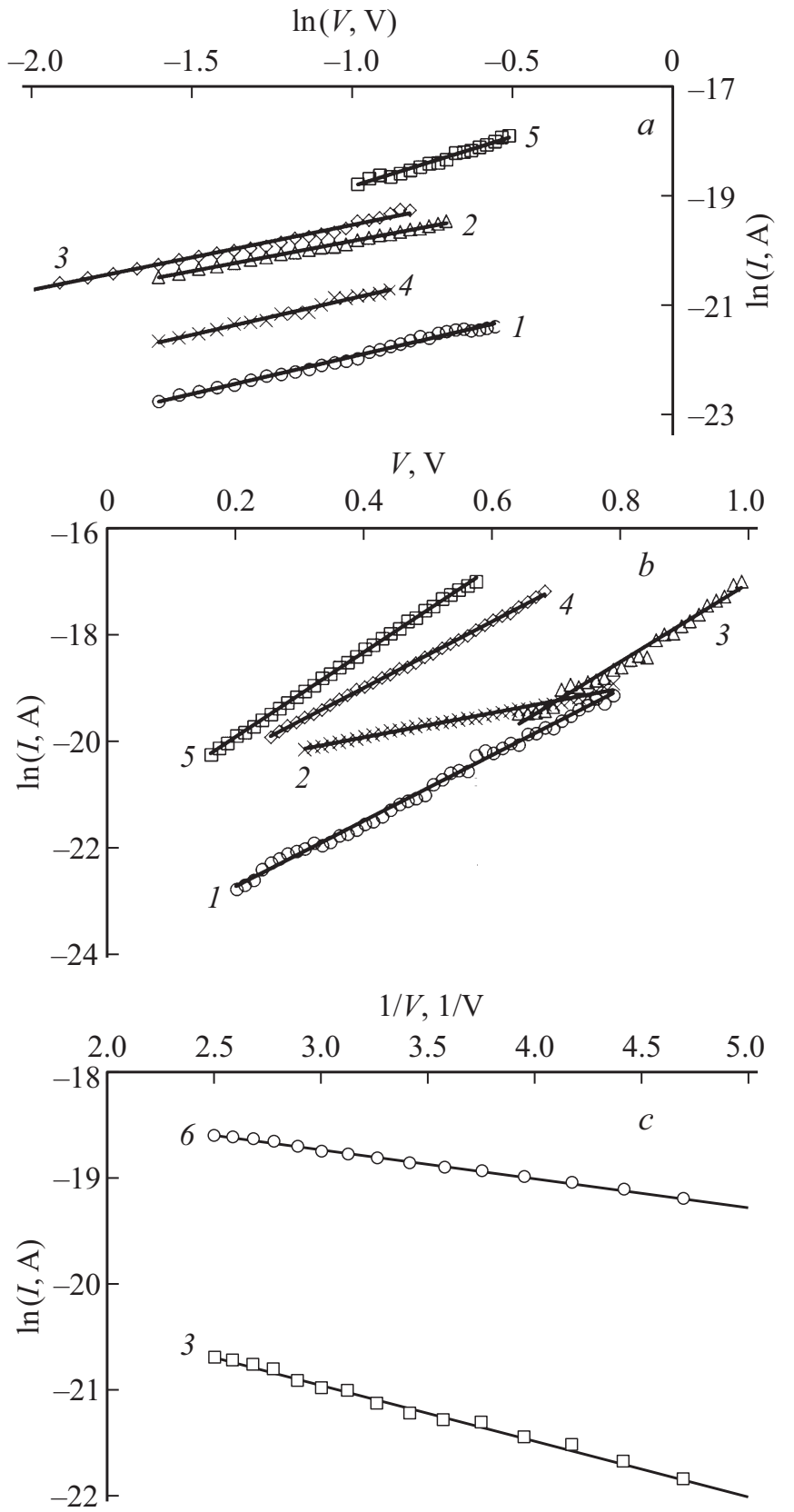

Рис. 2. ВАХ для одиночных КТ. Полярность на образце: $a-$ „Плюс“, $b$ и $c-$ „минус“. $1-\mathrm{CdSe}(\mathrm{CdS}), 2-\mathrm{PbS}(\mathrm{CdS})$, 3 - InSb, $4-\operatorname{InSb}(\operatorname{InP} / \mathrm{CdS})_{d 1}, 5-\mathrm{InSb}(\mathrm{InP} / \mathrm{CdS})_{d 2}, 6-\mathrm{PbS}$.

образце (эмиссия из зонда) ВАХ имела вид $I \sim A_{0} V^{a}$ (рис. 2,a). Полученные при этом средние по образцам значения параметров $A_{0}$ и $a$ приведены в таблице. Отклонения от средних значений не превышали $\pm 15 \%$. Анализ этих данных приводит к предположению о том, что транспорт электронов в этом случае происходит путем их инжекции из зонда через зазор $d_{0}$ в квантовую точку, термализации их состояний и пролета в ней. Степенная зависимость с показателем $1<a<2$ может свидетельствовать о явлении ограничения тока зарядом в объеме КТ [19]. При этом чем больше $a$, тем 
Сводные данные

\begin{tabular}{|c|c|c|c|c|c|c|c|c|c|c|c|c|}
\hline Полупроводник & $E_{g},{ } \mathrm{~B}$ & $m / m_{0}$ & $\varepsilon / \varepsilon_{0}$ & $d$, нм & $\Lambda$, HM & $\Lambda / d$ & $a$ & $A_{0}, 10^{-9}$ & $B_{0}, 1 / \mathrm{B}$ & $B_{0 T}, \mathrm{~B} / \mathrm{HMM}$ & $V_{\text {Qсои }}, \mathrm{B}$ & $V_{\text {Qmea }}, \mathrm{B}$ \\
\hline $\mathrm{CdSe}(\mathrm{CdS})$ & 1.75 & 0.13 & 9.5 & $2-3$ & 3 & 1 & 1.20 & 1.42 & 6.3 & & 0.6 & \\
\hline $\mathrm{PbS}(\mathrm{CdS})$ & \multirow[t]{2}{*}{0.41} & \multirow[t]{2}{*}{0.07} & \multirow[t]{2}{*}{170} & \multirow[t]{2}{*}{$4-5$} & \multirow[t]{2}{*}{8} & \multirow[t]{2}{*}{2} & \multirow[t]{2}{*}{1.10} & \multirow[t]{2}{*}{7.84} & 2.3 & & 0.02 & $0.1-0.5$ \\
\hline $\operatorname{PbS}(0)$ & & & & & & & & & & 0.21 & & \\
\hline $\operatorname{InSb}(0)$ & \multirow{3}{*}{0.18} & \multirow{3}{*}{0.02} & \multirow{3}{*}{$\begin{array}{c}18 \\
7-9\end{array}$} & $4-5$ & \multirow{3}{*}{$\begin{array}{c}20 \\
3\end{array}$} & \multirow{3}{*}{$\begin{array}{c}4 \\
4 \\
1.49\end{array}$} & 1.33 & 392 & & 0.29 & 0.2 & $0.5-1.0$ \\
\hline $\operatorname{InSb}(\operatorname{InP} / \mathrm{CdS})_{d 1}$ & & & & $4-5$ & & & 1.36 & 12.4 & 6.1 & & 0.2 & \\
\hline $\operatorname{InSb}(\operatorname{InP} / \mathrm{CdS})_{d 2}$ & & & & & & & 50.4 & 7.8 & & 0.1 & $1.0-1.5$ & \\
\hline
\end{tabular}

сильнее ограничение. На самом деле в этом случае происходит конкуренция двух явлений: пролет электрона с его ограничением объемным зарядом и перезарядка КТ-наноконденсатора. Степень влияния перезарядки характеризуют величины параметра $A_{0}$. Из сопоставлений величин $a$ и $A_{0}$ (см. таблицу) можно сделать заключение о том, что чем сильнее размерное квантование (больше $\Lambda / d$, см. таблицу) и чем больше емкость КТ (больше диэлектрическая проницаемость $\left.\varepsilon / \varepsilon_{0}\right)$, тем сильнее влияние пролета электрона и эффекта его ограничения по сравнению с перезарядкой.

Для полярности „минус“ на образце (эмиссия из КТ) для вариантов КТ с оболочкой ВАХ имела вид $I \sim \exp \left(B_{0} V\right)$ (рис. 2,b). Полученные средние по образцам значения параметра $B_{0}$ приведены в таблице. Отклонения от средних значений не превышали $\pm 15 \%$. По этим данным можно заключить, что транспорт электронов из КТ в зазор происходит благодаря их эмиссии. Вид эмиссии при этом может быть туннельной, полевой, тепловой - для его определения требуются температурные измерения, которые на используемой аппаратуре не было возможности провести. Для туннельной и полевой эмиссии, однако, должна выполняться зависимость вида $I \sim \exp \left(B_{0} / V\right)$, которая по BAX не наблюдалась. Поэтому можно предположить, что в данном случае механизм эмиссии был тепловым, когда должно быть: $B_{0} \sim q V_{Q 1}(k T)^{-1}$, где $V_{Q 1}$ - падение напряжения на КТ-наноконденсаторе емкостью $C_{Q 1}$. Можно считать, что доля напряжения $V_{Q 1} / V \cong C_{Q 0} / C_{Q 1} \sim d_{0} / d\left(\varepsilon / \varepsilon_{0}\right)$. Тогда (см. таблицу) для варианта $\mathrm{KT}-\mathrm{PbS}(\mathrm{CdS}) V_{Q 1} / V \sim 0.05$, а для остальных вариантов $-V_{Q 1} / V \sim 0.2$. Такую большую разницу можно объяснить большей величиной диэлектрической проницаемости сульфида свинца, что косвенно может свидетельствовать о возможной применимости модели диэлектрической поляризации к квантовым точкам.

Для вариантов КТ без оболочки (PbS, $\mathrm{InSb})$ ВАХ лучше аппроксимировалась зависимостью вида $I \sim \exp \left(B_{0 T} d_{0} / V\right)-$ рис. $1, c$. Это может означать, что для них более подходит модель туннелирования через локализованные уровни-состояния [17].

\section{2. ВАХ ансамблей КТ в планарной микроструктуре}

Исследования ВАХ ансамблей КТ в планарноторцевой микроструктуре показали схожесть с моделями электронного транспорта одиночных КТ [11]. Отличия, однако, заключаются в том, что при переносе электрона в последовательно-параллельной цепочке наночастиц ансамбля возможен токовый отбор пар КТ с минимальной величиной зазора для параллельной локальной части цепочки и максимальной - для последовательной. Это может оказать влияние на смену механизма в разных интервалах напряжения.

В определенных интервалах величин напряжения процесс лимитируется тем или иным механизмом последовательно-параллельного переноса электронов: при относительно небольших величинах напряжения $V$, когда затруднительна эмиссия - эмиссией электронов в межзеренный зазор, при больших $V$, когда эмиссия не лимитировала процесс - транспортом в самой квантовой точке. Исследование температурной зависимости эмиссионных участков ВАХ показало, что электронный транспорт определяется одним из лимитирующих процессов: термоэмиссией (KT-CdSe $(\mathrm{CdS})$, прямым (с дна зоны проводимости) туннелированием электронов $(\mathrm{KT}-\mathrm{PbS}(\mathrm{CdS})$, туннелированием через локализованные состояния InSb. При этом необходимо отметить, что первые два процесса характерны для квантовых точек с оболочкой.

Для проверки этого обстоятельства дополнительно проведены исследования на варианте сульфида свинца без оболочки - PbS. Измерения показали, что в этом случае $\mathrm{BAX}$ хорошо аппроксимируется зависимостью $I \sim \exp \left(B_{T} / V\right)$ со значениями $B_{T} \sim 200$ В. Используя модель электронного транспорта, развитую нами в работе [16], $-I / I_{0} \sim \exp \left(-B_{1 T} Z^{*} N / V\right)$, где $Z^{*}-$ средневзвешенный размер зазоров между наночастицами в линии тока, а $N-$ их количество. Полагая, что $B_{1 T}$ численно одинаково с $B_{0 T}$, и используя значение $B_{0 T} \sim 0.21$ (см. таблицу), получится для $Z^{*} N \sim 200 / 0.21=952$ нм. Это значение должно быть близко к величине межэлектродного размера в микроструктуре, в нашем случае (800-1000) HM. 


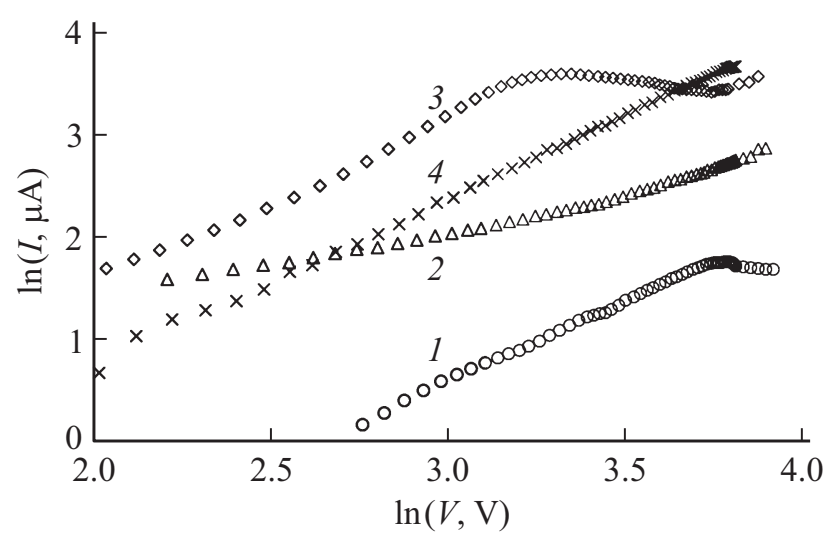

Рис. 3. ВАХ для ансамбля КТ в мультизеренной микроструктуре: $1,2-\operatorname{InSb}(2-\mathrm{c}$ ИК-засветкой $) ; 3,4-\mathrm{PbS}(4-\mathrm{c}$ УФ-засветкой).

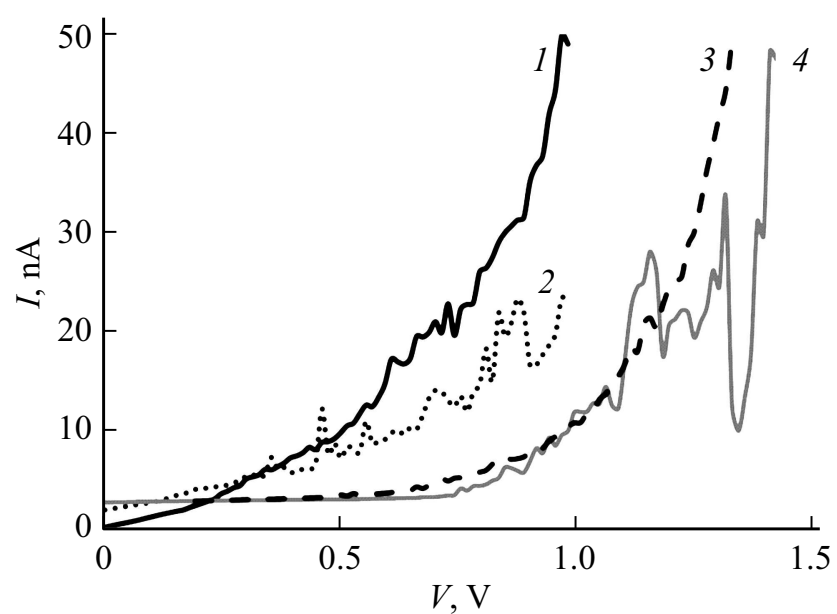

Рис. 4. Особенности ВАХ одиночных КТ: $1-\mathrm{CdSe}(\mathrm{CdS} / \mathrm{ZnS})$, $2-\mathrm{PbS}(\mathrm{CdS}), 3-\operatorname{InSb}(\mathrm{InP} / \mathrm{CdS}) d_{1}$ (белый свет), 4 $\operatorname{InSb}(\mathrm{InP} / \mathrm{CdS}) d_{1}$.

На рис. 3 приведены типичные ВАХ в билогарифмическом масштабе для интервала относительно больших величин напряжения (> 8.5 В), когда выполнялась зависимость $I \propto V^{m}$ при значениях $m \approx 1.5 \pm 0.2$. Этот результат свидетельствует о том, что ВАХ в этом интервале напряжений лимитируется транспортом электронов через квантовые точки с его ограничением локализованным зарядом. При этом независимо от различий (тип полупроводника, полярность, температура) аппроксимация давала практически одинаковые результаты, т. е. механизм транспорта был одинаково стабилен.

\section{3. Особенности электронного транспорта и BAX}

Главной особенностью электронного транспорта в квантовых точках является проявление кулоновской блокады в КТ и кулоновского провала на ВАХ [2-4]. Основные условия проявления кулоновской блокады - почти нулевое содержание электронов проводимости в КТ и соотношение энергии перезарядки наноконденсатора $-q(2 C)^{-1} \gg k T$. Условие по ограничению величины напряжения $V$ для проявления кулоновской блокады при туннелировании электронов сквозь наноемкостный барьер: $V \gg V_{Q}=q(n+1 / 2) C^{-1}$, где $V_{Q}$ - характерное напряжение проявления кулоновской блокады; $n$ число блокирующих электронов в КТ $[1,2,4]$.

В нашем случае для одиночных КТ оценки величин емкости КТ-шара и плоского наноконденсатора-зазора $C_{z}$ дают соотношение $C \gg C_{z}$, поэтому $V_{Q}$ для зазора много больше, чем для КТ. Важно также отметить, что проведенные измерения ВАХ во всех случаях при разных значениях $d_{0}$ не показали зависимости напряжения $V_{Q}$ в зонах особенностей от $d_{0}$. Из этих результатов следует, что наиболее влияющими для ограниченного электронного транспорта были сами квантовые точки. В таблице приведены рассчитанные значения $V_{Q c o и}$ и измеренные по ВАX $-V_{Q m e a}$. При этом в расчете $V_{Q c o u}$ принято $n=0$ из соображений, что произведение величин собственной концентрации носителей в использованных полупроводниках (для всех случаев $<10^{18} \mathrm{~cm}^{-3}$ ) на объем КТ $\left(\sim 10^{-20} \mathrm{~cm}^{3}\right)$ в наших случаях дает значения $n$, много меньшие единицы.

Примеры BAX с особенностью для одиночных КТ приведены на рис. 4. В этих зонах ВАХ наблюдались нестабильность тока и уменьшение его значений (провал). Сравнение $V_{Q c o u}$ и $V_{Q m e a ~}$ (см. таблицу) позволяет объяснить полученные в исследованиях результаты.

Для KT-CdSe $(\mathrm{CdS}), \mathrm{PbS}(\mathrm{CdS})$ и $\mathrm{InSb}$ хорошо выполняется условие $V_{Q m e a} \gg V_{Q c o u}$. В интервале наших режимных величин напряжения $\sim(0-1)$ В для КT-CdSe $(\mathrm{CdS})$ и InSb характерные особенности (провалы) не наблюдались, а для KT-PbS(CdS) наблюдались особенности на обеих ветвях ВАX.

Для КT-InSb $(\mathrm{InP} / \mathrm{CdS}) d_{1}$ и $\mathrm{InSb}(\mathrm{InP} / \mathrm{CdS}) d_{2}$ соотношение $V_{Q \text { теа }} \gg V_{Q \text { cou }}$ можно считать „избыточным“", что могло бы свидетельствовать о неравенстве нулю числа блокирующих электронов в КТ. Это возможно с учетом того, что источником таких электронов могут быть оборванные связи несоответствия решеток ядра и оболочки.

Хорошо проявляемые и объясняемые для одиночных КТ обсуждаемые здесь явления, не очевидны для их ансамблей, когда общий ток через нанослой складывается из его элементов в отдельных КТ. Тем не менее в экспериментах явно наблюдаются характерные участки BAX (рис. 3, кривые 1 и 3), с нестабильностью тока на них.

Другой интересной особенностью электронного транспорта в структурах с квантовыми точками является сильная зависимость эффекта от освещения. Засветка изменяет число неравновесных электронов (проводимости), изменяя $V_{Q}$ и тем самым срывая или усиливая кулоновскую блокаду, в результате чего в структуре происходит большое изменение величины тока (рис. 3 и 4). Воздействие белым и ближним инфракрасным (ИК) светом для всех вариантов приводило к 
увеличению тока (рис. 3 , кривая 2 и рис. 4 , кривая 3 ), что можно объяснить срывом ограничения тока, подобного кулоновской блокаде, за счет влияния возникающего в КТ большого числа неравновесных электронов проводимости, приводящих к смене механизма транспорта на фотопроводимость внутри КТ и фотоэмиссию между ними. Воздействие ультрафиолетом (УФ) влияло только на образцы с KT-PbS, приводя к аномальному эффекту уменьшению тока (рис. 3, кривая 4), что можно объяснить тем, что в КТ относительно широкозонного $\mathrm{PbS}$ возбуждаются высокоэнергетические электроны, способные „прилипать“ в состояния на ее границе и увеличивать тем самым блокирующий заряд, не изменяя механизма транспорта.

\section{4. Заключение}

Исследование ВАХ отдельных квантовых точек узкозонных полупроводников антимонида индия и сульфида свинца и их ансамблей в микроструктуре позволило считать, что в одноэлектронном режиме транспорт электронов в разных интервалах напряжения определяется одним из конкурирующих процессов: эмиссией из КТ, инжекцией в КТ и пролетом в ней с ограничением тока пространственным зарядом, перезарядкой наноконденсатора. ВАХ при этом определяется на отдельных участках одним из этих процессов при его лимитирующем действии. На начальном участке ВАХ лимитирующей является тепловая или туннельная эмиссия. C ростом напряжения лимитируют инжекция и ограничение зарядом в квантовой точке. При дальнейшем увеличении напряжения на ВАX наблюдались участки провала (нестабильности) тока. При этом, так же как и в случае кулоновской щели на ВАХ для квантовых точек, должно выполняться условие ограничения на величины энергии электрона, известное для квантовых точек как условие кулоновской блокады. Качественные и числовые сравнительные оценки позволяют считать, что в реальной (приборной) структуре сегрегированного множества квантовых точек также могут наблюдаться одноэлектронный транспорт и ограничение тока, подобное кулоновской блокаде. Засветка срывает или усиливает эффект, приводя к увеличению или уменьшению тока в зависимости от спектра возбуждающего излучения.

\section{Финансирование работы}

Исследование выполнено при финансовой поддержке РФФИ в рамках научного проекта 19-07-00087-а.

\section{Благодарности}

Авторы благодарны С.В. Дежурову и О.Ю. Цветковой за изготовление образцов квантовых точек, д.Х.н. Д.В. Крыльскому за полезные советы.

\section{Конфликт интересов}

Авторы заявляют, что у них нет конфликта интересов.

\section{Список литературы}

[1] Е.С. Солдатов, В.В. Колесов. Радиоэлектроника. Наносистемы. Информационные технологии, 4 (2), 71 (2012).

[2] A. Kurzmann, P. Stegmann, J. Kerski, R. Schott, A. Ludwig, A.D. Wieck, J. König, A. Lorke, M. Geller. Phys. Rev. Lett., 122, 247403 (2019).

[3] А.А. Щука. Наноэлектроника (М.,Физматкнига, 2007).

[4] Y. Imry. Introduction to Mesoscopic Physics (N. Y., Oxford, 1997).

[5] В.О. Залунин. Автореф. канд. дис. (М., МГУ, 2012).

[6] В.В .Шорохов. Автореф. канд. дис. (М., МГУ, 2007).

[7] C. Wasshuber. Diss. (Wien, Technische Universität Wien, 1997).

[8] С.А. Дагесян, В.В. Шорохов, Д.Е. Преснов, Е.С. Солдатов, А.С. Трифонов, В.А. Крупенин, О.В. Снигирев. Вестн. Моск. ун-та, 3 (5), 32 (2017).

[9] М.В. Максимов, А.Е. Жуков. [Электронный ресурс] URL http://wmw-magazine.ru/uploads/volumes/11/maksimov.pdf

[10] Н.Д. Жуков, М.В. Гавриков, Д.В. Крыльский. Письма ЖТФ, 46 (17), 47 (2020).

[11] Н.Д. Жуков, И.Т. Ягудин, Н.П. Абаньшин, Д.С. Мосияш. Письма ЖТФ, 46 (21), 40 (2020).

[12] Д.В. Крыльский, Н.Д. Жуков. Письма ЖТФ, 45 (16), 10 (2019).

[13] С.В. Дежуров, А.Ю. Трифонов, М.В. Ловыгин, А.В. Рыбакова, Д.В. Крыльский. Росс. нанотехнологии, 11 (5), 54 (2016).

[14] V.F. Kabanov, Y.E. Pereverzev, I.A. Gorbachev, M.V. Gavrikov, E.G. Glukhovskoy. 1st International Conference on Advanced Energy Materials, AEM 2016 (Guildford, England, 2016) v. 1, p. 40.

[15] С.А. Рыков. Сканирующая зондовая микроскопия полупроводниковых материалов и нано-структур (СПб., Наука, 2001).

[16] Н.Д. Жуков, Д.С. Мосияш, И.В. Синёв, А.А. Хазанов, А.В. Смирнов, И.В. Лапшин. Письма ЖТФ, 43 (24), 72 (2017).

[17] Н.Д. Жуков, В.Ф. Кабанов, А.И. Михайлов, Д.С. Мосияш, А.А. Хазанов, М.И. Шишкин. ФТП, 52 (1), 83 (2018).

[18] Т.В. Бланк, Ю.А. Гольдберг. ФТП, 41 (11), 1281 (2007).

[19] Н.Д. Жуков, Е.Г. Глуховской, А.А. Хазанов. ФТП, 50 (6), 772 (2016).

Редактор Г.А. Оганесян 


\section{Single-electron emission-injection \\ transport in a microstructure with colloidal quantum dots of narrow-gap \\ semiconductors}

N.D. Zhukov' ${ }^{1}$, M.V. Gavrikov ${ }^{1,2}$, V.F. Kabanov' ${ }^{2}$, I.T. Yagudin ${ }^{1}$

${ }^{1}$ LLC „Research and production enterprise Volga“, 410033 Saratov, Russia

${ }^{2}$ Saratov State University, 410012 Saratov, Russia

Abstract By approximating the current-voltage characteristics of colloidal quantum dots of narrow-gap semiconductors InSb and $\mathrm{PbS}$, it is shown that in the one-electron mode, electron transport in different voltage intervals is determined by one of the competing processes - emission from a quantum dot, injection into it and transport through it with current limitation by space charge. At voltages above $0.5 \mathrm{~V}$, for single quantum dot on the currentvoltage characteristics, regions of instability and current dip similar to the Coulomb gap were observed. Qualitative and numerical comparative estimates suggest that one-electron transport and current limitation similar to the Coulomb blockade are observed in the structure of a segregated set of quantum dots. Exposure to light when measuring the current-voltage characteristics breaks or enhances the Coulomb blockade, increasing or decreasing the current, depending on the spectrum of the exciting radiation. 